\title{
The Longitudinal Evolution of Cerebral Blood Flow Regulation after Acute Ischaemic Stroke
}

\author{
Angela S.M. Salinet ${ }^{a}$ Ronney B. Panerai ${ }^{a, b}$ Thompson G. Robinson ${ }^{a, b}$ \\ ${ }^{a}$ Department of Cardiovascular Sciences, University of Leicester and ${ }^{b}$ National Institutes \\ for Health Research, Biomedical Research Unit in Cardiovascular Sciences, Clinical Sciences \\ Wing, Glenfield Hospital, Leicester, UK
}

\section{Key Words}

Cerebral haemodynamics · Neurovascular coupling · Cerebral blood flow · Neural activation ·

Acute ischaemic stroke

\begin{abstract}
Background: Acute stroke is known to impair cerebral blood flow (CBF) regulation, but the longitudinal changes of these effects have been poorly reported. The main CBF regulatory mechanisms [cerebral autoregulation (CA) and neurovascular coupling (NVC)] were assessed over 3 months after acute ischaemic stroke. Methods: Recordings of CBF velocity (CBFv), blood pressure (BP), and end-tidal $\mathrm{CO}_{2}$ were performed during 5 min baseline and 1 min passive movement of the elbow. Stroke patients were assessed $<72 \mathrm{~h}$ of stroke onset, and at 2 weeks, 1 and 3 months after stroke. Results: Fifteen acute stroke subjects underwent all 4 sessions and were compared to 22 control subjects. Baseline recordings revealed a significantly lower CBFv in the affected hemisphere within $72 \mathrm{~h}$ after stroke compared to controls $(p=0.02)$ and a reduction in CA index most marked at 2 weeks $(p=0.009)$. CBFv rise in response to passive arm movement was decreased bilaterally after stroke, particularly in the affected hemisphere $(p<0.01)$. Both alterations in CA and NVC returned to control levels during recovery. Conclusion: The major novel finding of this study was that both CA and NVC regulatory mechanisms deteriorated initially following stroke onset, but returned to control levels during the recovery period. These findings are relevant to guide the timing of interventions to manipulate BP and potentially for the impact of intensive rehabilitation strategies that may precipitate acute physiological perturbations but require further exploration in a larger population that better reflects the heterogeneity of stroke. Further, they will also enable the potential influence of stroke subtype to be investigated.




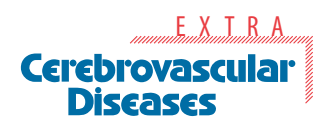

\begin{tabular}{l|l}
\hline Cerebrovasc Dis Extra 2014;4:186-197 \\
\hline DOI: 10.1159/000366017 & $\begin{array}{l}\text { C 2014 S. Karger AG, Basel } \\
\text { www.karger.com/cee }\end{array}$ \\
\hline Salinet et al.: The Longitudinal Evolution of Cerebral Blood Flow Regulation after Acute
\end{tabular} Salinet et al.: The
Ischaemic Stroke

\section{Introduction}

Abnormalities in cerebral haemodynamics, such as changes in local perfusion pressure and vascular integrity [1], are important pathophysiological elements in ischaemic stroke. The inadequate cerebral blood flow (CBF) supply may produce damage to neurons or glial cells beyond that caused by the initial ischaemia [1] due to either hypo- or hyperperfusion. The homeostatic balance in the human brain is regulated by two dynamic mechanisms called cerebral autoregulation (CA) and neurovascular coupling (NVC). In the normal brain, CA is a mechanism responsible for maintaining $\mathrm{CBF}$ relatively constant over a wide range of blood pressure (BP) variations. NVC refers to a cascade of events triggered by an increase in neuronal activity leading to an increase in CBF $[2,3]$. Both mechanisms are challenged after acute ischaemic stroke [4-6].

Although previous studies have contributed greatly to our understanding of brain reorganization after cerebral ischemia, the influence of impaired haemodynamics on the improvement of clinical brain function over time is still unclear [4-7]. Therefore, there is an urgent need to investigate the pattern of cerebral haemodynamic changes over time, with greater statistical power than is possible from cross-sectional studies. Previous longitudinal investigations of cerebral activity and/or cerebral regulation have been limited to two (the majority of studies) or three separate observations [6-14]. Epidemiological studies have suggested that the greatest amount of recovery occurs during the first 3 months after stroke onset [15].

Therefore, this prospective study aimed to investigate the temporal evolution of the changes in CBF regulation, assessing both CA and NVC mechanisms, within $72 \mathrm{~h}$ of acute ischaemic stroke onset, and at 3 additional time points over a 3-month recovery period. The first question addressed in this study was whether CBF regulation is impaired acutely within 72 $\mathrm{h}$ of stroke onset. A second question evaluated what the longitudinal changes of CBF regulation are during the recovery period, particularly the CBF velocity (CBFv) response to a passive motor paradigm.

\section{Methods}

\section{Participants}

Study participants were recruited from the Hyperacute Stroke Unit at the University Hospitals of Leicester NHS Trust. Inclusion criteria were as follows: (1) first episode of ischaemic stroke, confirmed by a single acute ischaemic brain lesion on MRI and/or CT, and (2) within $72 \mathrm{~h}$ of symptom onset. Exclusion criteria comprised: (1) history of neurological disorder; (2) atrial fibrillation, and (3) severe cognitive impairment that might prevent completion of the study. The Nottingham Research Ethics Committee 1, UK approved the research protocol (ref.: 11/EM/0016), and all study subjects gave informed consent before any study assessments.

\section{Experimental Design}

The study protocols were planned as 3-month longitudinal investigations, during which patients underwent neurological and transcranial Doppler (TCD) assessments on 4 occasions after infarction occurred. Stroke participants were assessed in the acute phase $(<72 \mathrm{~h})$ and at follow-up ( 2 weeks, and 1 and 3 months). A cross-sectional enrolment design also included a group of right-handed healthy controls matched for age and sex; handedness was established by the Edinburgh Inventory [16]. Neurological impairment and dependency were assessed utilizing the National Institutes of Health Stroke Scale [17] and the modified Rankin Scale [18], respectively. 
Salinet et al.: The Longitudinal Evolution of Cerebral Blood Flow Regulation after Acute Ischaemic Stroke

\section{Data Acquisition}

The study was conducted in a quiet research laboratory with participants lying in a comfortable supine position. CBFv in the middle cerebral artery (MCA) was continuously assessed by TCD ultrasound (Viasys Companion III; Viasys Healthcare) using a dual 2-MHz transducer fitted to a head frame. BP and heart rate were continuously recorded by a Finapres device (Ohmeda 2300; Finapres, Louisville, Colo., USA) and 3-lead electrocardiogram, respectively. End-tidal $\mathrm{CO}_{2}\left(\mathrm{EtCO}_{2}\right)$ was monitored using a capnograph (Capnocheck Plus) with nasal sampling. All participants had abstained from caffeine, alcohol and nicotine for $2 \mathrm{~h}$ before the measurement.

After 15 min of stabilization, a 5-min baseline recording was taken, and the stimulation paradigm was then performed twice. This included a 90 -second baseline phase, the paradigm performed over $60 \mathrm{~s}$, and a 90-second recovery phase. The paradigm consisted of $1 \mathrm{~min}$ passive flexion and extension of the elbow, as described previously [19-21]. The rate of opposition was driven by a metronome $(1 \mathrm{~Hz})$, an electrical signal which marked the start and finish of the manoeuvre. Movement was performed only on the affected side in the stroke group and the dominant side (right) in controls.

\section{Data Analysis}

Data were simultaneously recorded onto a data acquisition system (Physidas, Department of Medical Physics, University Hospitals of Leicester) at a sampling rate of 500 samples/s. CBFv channels were filtered at $20 \mathrm{~Hz}$ (zero-phase eighth-order Butterworth), R-R interval was obtained using electrocardiogram, and mean $\mathrm{BP}, \mathrm{CBFv}$ and $\mathrm{EtCO}_{2}$ were calculated for each cardiac cycle beat. Beat-to-beat data were spline interpolated and resampled at $5 \mathrm{~Hz}$ to produce signals with a uniform time base. The manoeuvre showing the largest CBFv response was chosen to represent the participant's response [19]. Mean CBFv, heart rate, BP and $\mathrm{EtCO}_{2}$ values were extracted from the 30 s preceding the paradigm for baseline comparisons. The beginning of stimulation was used as the point of synchronization to obtain population mean and SD curves for each variable and each hemisphere.

The CBFv step response to the BP input was computed on the 5-min baseline measurement, after a period of $100 \mathrm{~s}$ of stabilization, as previously described [22]. A fast Fourier transform was applied to the data, and the cross- and auto-spectra were estimated using the Welch method. The transfer function of the BP-CBFv dynamic relationship was then calculated with BP selected as the input, and right then left CBFv as the output variables. An inverse fast Fourier transform was then applied to the complex transfer function, converting data back into the time domain, to calculate the CBFv step response [22]. The autoregulatory index (ARI) was assigned to each recording by using the best least-squares fit between the CBFv step response and one of the 10 model ARI curves proposed by Tiecks et al. [23]. Values of ARI $=0$ represent absence of CA, while values of ARI $=9$ correspond to best-observed autoregulation. ARI was calculated for each subject for both hemispheres at baseline.

Using a standard protocol, responses obtained from the affected hemisphere (AH) in stroke were compared to the responses in the dominant hemisphere in the right-handed control population, whereas the unaffected hemisphere (UH) results were compared to the right hemisphere in controls [21].

\section{Statistical Analysis}

Baseline CBFv values ( 5 min recording at rest and $30 \mathrm{~s}$ preceding the exercise performance) were compared using two-way ANOVA with group (control and patient) as the between factor and side of recording (right, left, $\mathrm{AH}, \mathrm{UH}$ ) as the within factor. A t test for independent samples was used to compare baseline values of heart rate, $\mathrm{BP}$ and $\mathrm{EtCO}_{2}$ between control and acute stroke participants (session 1). To compare the effect of the paradigms on 


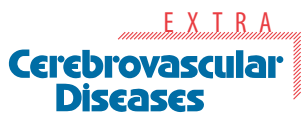

Table 1. Participant characteristics

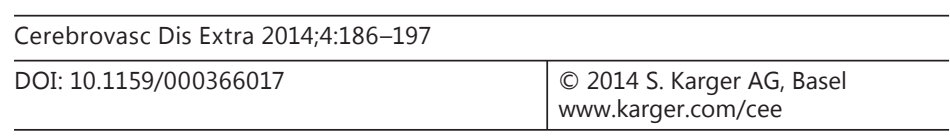

Salinet et al.: The Longitudinal Evolution of Cerebral Blood Flow Regulation after Acute Ischaemic Stroke

\begin{tabular}{|c|c|c|}
\hline Variables & Stroke $(n=15)$ & Controls $(n=22)$ \\
\hline Age $^{a}$, years & $62.4 \pm 9.0$ & $62.2 \pm 7.5$ \\
\hline Male, $\mathrm{n}$ & 12 & 16 \\
\hline Edinburgh Inventorya , \% & $84.4 \pm 6.2$ & $89.9 \pm 3.6$ \\
\hline \multicolumn{3}{|l|}{ Baseline medication } \\
\hline Antihypertensive & 4 & \\
\hline Statin & 1 & \\
\hline Hypertension & 5 & - \\
\hline Myocardial infarction & 2 & - \\
\hline Thrombolysis & 2 & - \\
\hline Diabetes mellitus & 0 & - \\
\hline Hypercholesterolaemia & 1 & - \\
\hline \multicolumn{3}{|l|}{ Ipsilateral ICA stenosis } \\
\hline$>50 \%$ & $1^{\mathrm{b}}$ & - \\
\hline $30-49 \%$ & $1^{\mathrm{b}}$ & - \\
\hline$<30 \%$ & 1 & - \\
\hline \multicolumn{3}{|l|}{ Contralateral ICA stenosis } \\
\hline $30-49 \%$ & 2 & - \\
\hline \multirow{2}{*}{\multicolumn{3}{|c|}{ NIHSS score ${ }^{\mathrm{a}}$}} \\
\hline & & \\
\hline Admission & $7.8 \pm 4.8$ & - \\
\hline Session 1 & $3.4 \pm 1.9$ & - \\
\hline Session 2 & $1.9 \pm 1.8$ & - \\
\hline Session 3 & $1.2 \pm 1.6$ & - \\
\hline Session 4 & $0.7 \pm 1.3$ & - \\
\hline \multicolumn{3}{|l|}{ mRS score ${ }^{a}$} \\
\hline Session 1 & $2.1 \pm 1.2$ & - \\
\hline Session 4 & $0.4 \pm 0.5$ & - \\
\hline \multicolumn{3}{|c|}{$\begin{array}{l}\text { ICA = Internal carotid artery; NIHSS = National Institutes of Health } \\
\text { Stroke Scale; mRS = modified Rankin Scale. } \\
\text { a Figures are given as means } \pm \text { SD. } \\
\text { b Ipsilateral and contralateral ICA stenosis. }\end{array}$} \\
\hline
\end{tabular}




\section{Cerebrovascular \\ Diseases}

Fig. 1. Mean values $( \pm 1$ standard error) of ARI for healthy controls $(\mathrm{R}, \mathrm{L})$ and the stroke group. The stroke hemispheres are represented by affected (A) and unaffected (U), respectively, followed by the session number corresponding to $<72 \mathrm{~h}(1), 2$ weeks (2), 1 month (3) and 3 months (4) of follow-up.

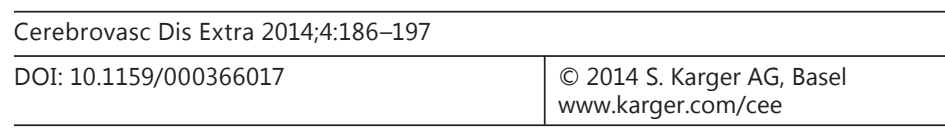

Salinet et al.: The Longitudinal Evolution of Cerebral Blood Flow Regulation after Acute Ischaemic Stroke

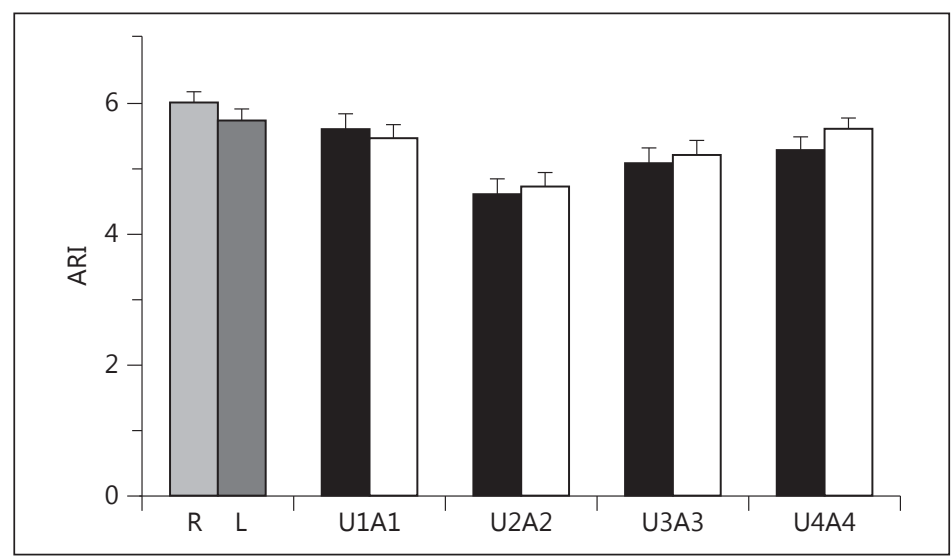

Table 2. Mean \pm SD values for cerebral and peripheral haemodynamic variables during the 5 min baseline and the $30 \mathrm{~s}$ preceding the passive paradigm

\begin{tabular}{|c|c|c|c|c|c|c|c|}
\hline \multirow[t]{2}{*}{ Variables } & \multicolumn{2}{|c|}{$\mathrm{CBFv}, \mathrm{cm} \cdot \mathrm{s}^{-1}$} & \multirow[t]{2}{*}{$\mathrm{BP}, \mathrm{mm} \mathrm{Hg}$} & \multirow[t]{2}{*}{$\mathrm{HR}, \mathrm{bpm}$} & \multirow{2}{*}{$\begin{array}{l}\mathrm{EtCO}_{2} \\
\mathrm{~mm} \mathrm{Hg}\end{array}$} & \multicolumn{2}{|l|}{ ARI } \\
\hline & $\begin{array}{l}\text { right/ } \\
\text { unaffected }\end{array}$ & $\begin{array}{l}\text { left/ } \\
\text { affected }\end{array}$ & & & & $\begin{array}{l}\text { right/ } \\
\text { unaffected }\end{array}$ & $\begin{array}{l}\text { left/ } \\
\text { affected }\end{array}$ \\
\hline \multicolumn{8}{|c|}{5 min baseline } \\
\hline Control & $48.9 \pm 4.9$ & $50.7 \pm 5.6$ & $87.2 \pm 11.0$ & $58.8 \pm 8.0$ & $37.7 \pm 3.2$ & $6.0 \pm 0.9$ & $5.7 \pm 0.9$ \\
\hline Session 1 & $49.5 \pm 10.1$ & $45.4 \pm 6.9^{*}$ & $91.9 \pm 8.7^{*}$ & $65.3 \pm 7.7^{*}$ & $35.1 \pm 2.6^{*}$ & $5.6 \pm 1.3$ & $5.5 \pm 1.3$ \\
\hline Session 2 & $43.9 \pm 9.9$ & $48.3 \pm 8.6$ & $93.7 \pm 8.4$ & $68.5 \pm 10.3$ & $35.6 \pm 5.3$ & $4.6 \pm 1.3$ & $4.7 \pm 1.2$ \\
\hline Session 3 & $46.0 \pm 6.1$ & $48.8 \pm 9.9$ & $93.8 \pm 9.6$ & $64.3 \pm 8.8$ & $34.9 \pm 2.1$ & $5.1 \pm 1.3$ & $5.2 \pm 1.8$ \\
\hline Session 4 & $47.9 \pm 9.5$ & $49.0 \pm 9.8$ & $94.8 \pm 7.6$ & $67.0 \pm 9.3$ & $35.4 \pm 2.1$ & $5.3 \pm 1.0$ & $5.6 \pm 1.0$ \\
\hline \multicolumn{8}{|c|}{30 s before paradigm } \\
\hline Control & $49.5 \pm 5.5$ & $52.9 \pm 5.0$ & $87.2 \pm 11.0$ & $59.1 \pm 7.6$ & $39.1 \pm 3.1$ & & \\
\hline Session 1 & $48.8 \pm 8.0$ & $47.1 \pm 9.2^{*}$ & $93.6 \pm 8.0^{*}$ & $67.9 \pm 9.6^{*}$ & $34.8 \pm 2.3^{*}$ & & \\
\hline Session 2 & $44.3 \pm 9.6$ & $47.3 \pm 8.8$ & $91.5 \pm 7.6$ & $64.9 \pm 9.7$ & $35.6 \pm 3.4$ & & \\
\hline Session 3 & $45.7 \pm 8.0$ & $48.1 \pm 9.6$ & $91.7 \pm 7.5$ & $65.4 \pm 10.0$ & $35.8 \pm 3.2$ & & \\
\hline Session 4 & $48.6 \pm 8.9$ & $49.2 \pm 8.9$ & $91.7 \pm 7.6$ & $64.6 \pm 10.3$ & $36.1 \pm 2.3$ & & \\
\hline
\end{tabular}

HR $=$ Heart rate. ${ }^{*} \mathrm{p}<0.02$ (Tukey's post hoc test) for the comparison between stroke (session 1) and control.

The acute assessment (session 1) was undertaken a mean (SD) of $36.6 \mathrm{~h}$ (15.0) after the stroke onset, whereas the subacute (session 2) and chronic phases (sessions 3 and 4) were at 14.0 (1.0), 30.7 (1.2) and 94.2 days (0.7) after stroke, respectively.

\section{Baseline Recordings}

Resting values of the recorded parameters during the 5-min and 30-second baseline periods are shown in table 2. Though bilateral CBFv was lower in the stroke group during session $1(<72 \mathrm{~h}$ ) than in the control group (two-way ANOVA group interaction, $\mathrm{p}=0.03$ ), only the AH CBFv was statistically lower than the left-hemisphere CBFv in controls (Tukey's $\mathrm{p}=$ 0.02). A gradual increase in the AH CBFv was seen on follow-up. On the other hand, the UH CBFv dropped at session 2 (decrease seen in 11/15 patients) which coincided with the drop in ARI values (table 2; fig. 1). However, no statistical difference between assessments was 


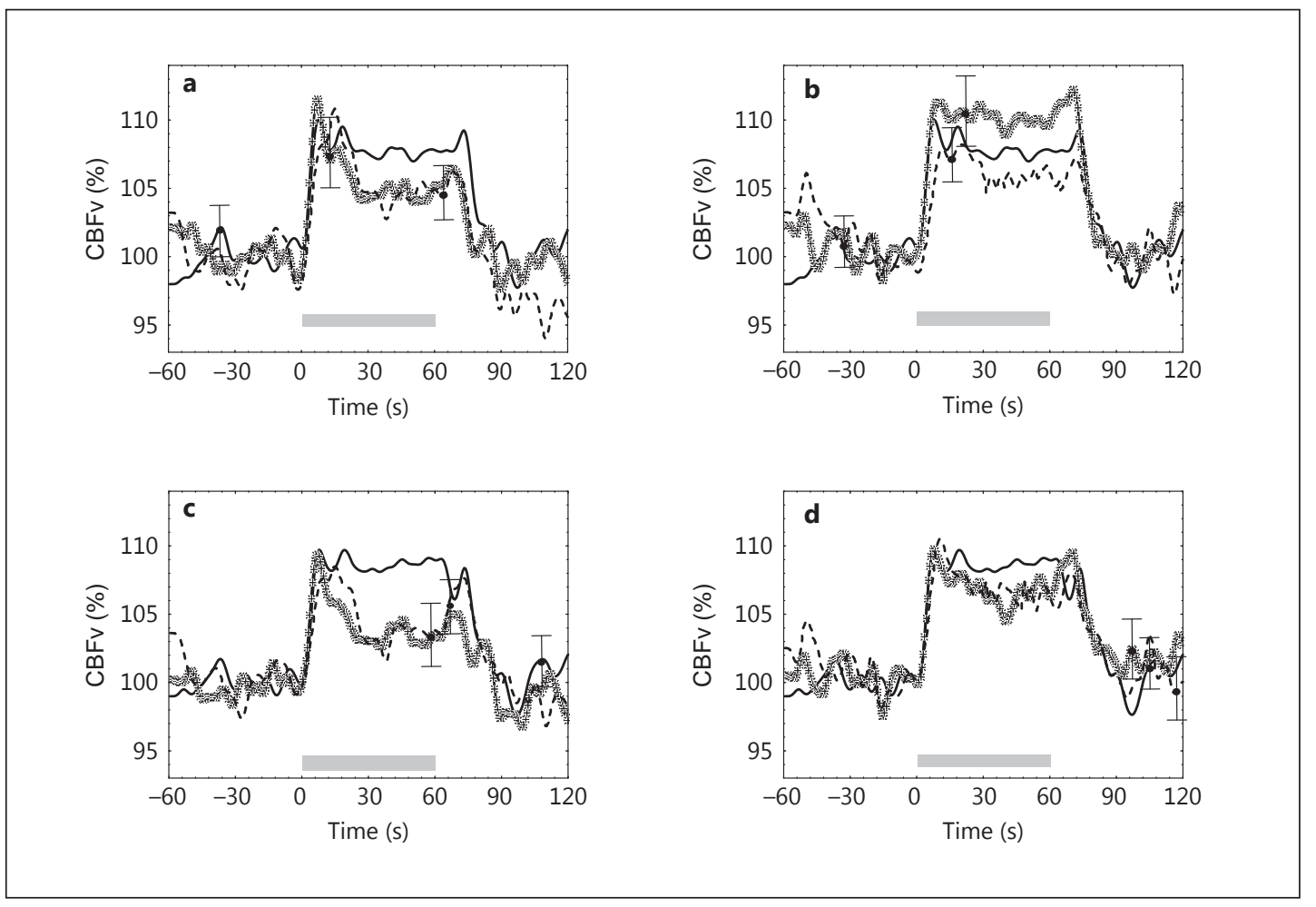

Fig. 2. Population averages of CBFv in healthy controls and stroke at acute, subacute and chronic ( 1 and 3 months) time points during passive arm movement (duration indicated by grey bar). a Response to the right hemisphere of control subjects (continuous line) and to the UH in stroke patients during the acute (continuous line + symbol) and subacute (dashed line) phases. b Response to the right hemisphere of control subjects (continuous line) and to the UH in stroke patients during the chronic phases (1 month, continuous line + symbol; 3 months, dashed line). c Response to the left hemisphere of control subjects (continuous line) and to the $\mathrm{AH}$ in stroke patients during the acute (continuous line + symbol) and subacute (dashed line) phases. d Response to the left hemisphere of control subjects (continuous line) and to the AH in stroke patients during the chronic phases ( 1 month, continuous line + symbol; 3 months, dashed line). For clarity, only the largest \pm 1 standard error is represented at the point of occurrence.

found in the repeated-measures ANOVA. Stroke participants were consistently more hypertensive $(p=0.03)$, tachycardic $(p=0.009)$ and hypocapnic $(p=0.003)$ than controls showing the same pattern over time. Similar to the 5 -min baseline values, the resting values of the affected $\mathrm{CBFv}, \mathrm{BP}$, heart rate and $\mathrm{EtCO}_{2}$ extracted from the $30 \mathrm{~s}$ preceding the passive elbow movement were significantly different between the stroke (session 1) and control groups (table 2).

\section{Passive Paradigm}

A sharp bilateral increase in $\mathrm{CBFv}$ in response to the passive paradigm was observed in all subjects (fig. 2), but the rise was less pronounced in the stroke group except for the UH in session 3 (table 3). Tukey's post hoc test revealed differences between left hemisphere and $\mathrm{AH}(\mathrm{p}=0.001)$, and between right hemisphere and UH $(\mathrm{p}=0.05)$. Table 3 and figure 3 illustrate the mean values of $\mathrm{CBFv}, \mathrm{BP}$, heart rate and $\mathrm{EtCO}_{2}$ for the first $80 \mathrm{~s}$ of the passive paradigm. 


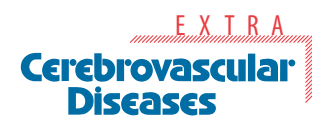

\begin{tabular}{l|l}
\hline \multicolumn{2}{l}{ Cerebrovasc Dis Extra 2014;4:186-197 } \\
\hline DOI: $10.1159 / 000366017$ & $\begin{array}{l}\text { C 2014 S. Karger AG, Basel } \\
\text { www.karger.com/cee }\end{array}$ \\
\hline Salinet et al.: The Longitudinal Evolution of Cerebral Blood Flow Regulation after Acute
\end{tabular} Salinet et al.: The
Ischaemic Stroke

Table 3. Mean $\pm \mathrm{SD}$ values of the $\mathrm{CBFv}$ response, $\mathrm{BP}$, heart rate and $\mathrm{EtCO}_{2}$ responses to passive paradigm for patients (sessions 1-4) and controls

\begin{tabular}{llllll}
\hline Variables & $\mathrm{CBFv}, \%$ & \multirow{2}{*}{$\mathrm{BP}, \mathrm{mm} \mathrm{Hg}$} & $\mathrm{HR}, \mathrm{bpm}$ & $\mathrm{EtCO}_{2}, \mathrm{~mm} \mathrm{Hg}$ \\
\cline { 2 - 3 } & right/unaffected & left/affected & & \\
\hline Control & $107.8 \pm 6.2$ & $108.3 \pm 5.5$ & $90.5 \pm 10.5$ & $60.7 \pm 8.0$ & $38.8 \pm 3.0$ \\
Session 1 & $105.4 \pm 5.3$ & $104.1 \pm 4.9^{*}$ & $94.1 \pm 9.3$ & $69.8 \pm 10.9$ & $34.6 \pm 2.4$ \\
Session 2 & $105.5 \pm 4.3$ & $104.9 \pm 2.9$ & $92.2 \pm 10.1$ & $69.4 \pm 11.7$ & $35.5 \pm 2.7$ \\
Session 3 & $110.0 \pm 6.9^{¥}$ & $107.0 \pm 4.7$ & $91.4 \pm 8.9$ & $64.8 \pm 10.4$ & $35.9 \pm 3.2$ \\
Session 4 & $105.9 \pm 5.8$ & $106.4 \pm 4.8$ & $90.9 \pm 8.1$ & $64.3 \pm 12.8$ & $36.3 \pm 2.3$ \\
\hline
\end{tabular}

HR $=$ Heart rate. ${ }^{*} \mathrm{p}<0.01$ (Tukey's post hoc test) for the differences between control (left hemisphere) and stroke (AH; session 1) groups. ${ }^{¥} \mathrm{p}<0.04$ (Tukey’s post hoc test; not significant after Bonferroni correction) between sessions 1 and 3, between sessions 2 and 3, and between sessions 3 and 4 .

\section{Discussion}

\section{Main Findings}

This study is the first to report the evolution of serial TCD assessments of CA and NVC mechanisms in the same stroke population followed over 3 months after stroke. In agreement with functional neural activation studies [10], the longitudinal responses of the CBFv to neural activation showed a triphasic trend from the acute to the chronic stage. The initially reduced bilateral $\mathrm{CBFv}$ response was followed by a $\mathrm{CBFv}$ increase in the $\mathrm{UH}$ after a month, which progressively approximated to the normal CBFv response pattern by 3 months. Other longitudinal studies have also shown signs of the progressive functional improvement of the CBF response or neural activation pattern in the stroke $\mathrm{AH}[8,9,12]$. This observation is also consistent with findings from longitudinal studies in a rat stroke model in which functional improvement was accompanied by reactivation of the affected cortex over time [25].

In recent years, several investigators have found evidence for reorganization of the neural network after stroke, not only in the AH but also in the UH [12, 26, 27]. Moreover, overactivation of certain areas in the UH in the subacute phase was associated with clinical recovery $[14,28]$. This is in line with our results, as the studied population all had a good recovery within weeks of stroke onset (table 1) and the improved UH CBFv response was seen in the majority of participants by 1 month. The mechanisms of involvement of areas located in the UH seem to be effective not only in recovery from motor deficits $[29,30]$, but even in the favourable recovery of aphasia [31,32].

Though CA impairment was not found acutely, a significant reduction in ARI was seen by 2 weeks, coinciding with a drop in baseline CBFv in the UH. The intra-individual time course of dynamic autoregulatory disturbance has not been extensively studied in humans from the first days after cerebral ischaemia. Previous studies analysing the CA in acute stroke found an impairment of dynamic CA $[6,13,33]$ that lasted for at least $1-2$ weeks [13]. This was associated with poor functional recovery [34].

There is always the possibility of the pressure-velocity relationship being strongly affected by changes in other physiological parameters like sympathetic activation, cerebral venous pressure, breathing frequency, and $\mathrm{CO}_{2}$ levels [35]. Because $\mathrm{CO}_{2}$ has a marked influence on CBFv and also on autoregulation itself, it is possible that the hypocapnia observed in the stroke patients has masked a modest CA impairment acutely. Further studies are needed to address this specific question. 


\section{Cerebrovascular' \\ Diseases}

Fig. 3. Mean values and standard error of BP (a), heart rate (b) and $\mathrm{EtCO}_{2}$ (c) for healthy controls and stroke group with the 4 sessions represented by S1 (<72 h), S2 (2 weeks), S3 (1 month) and S4 (3 months), respectively.

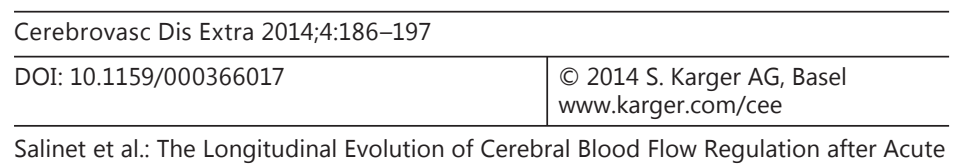

Salinet et al.: The Longitudinal Evolution of Cerebral Blood Flow Regulation after Acute Ischaemic Stroke
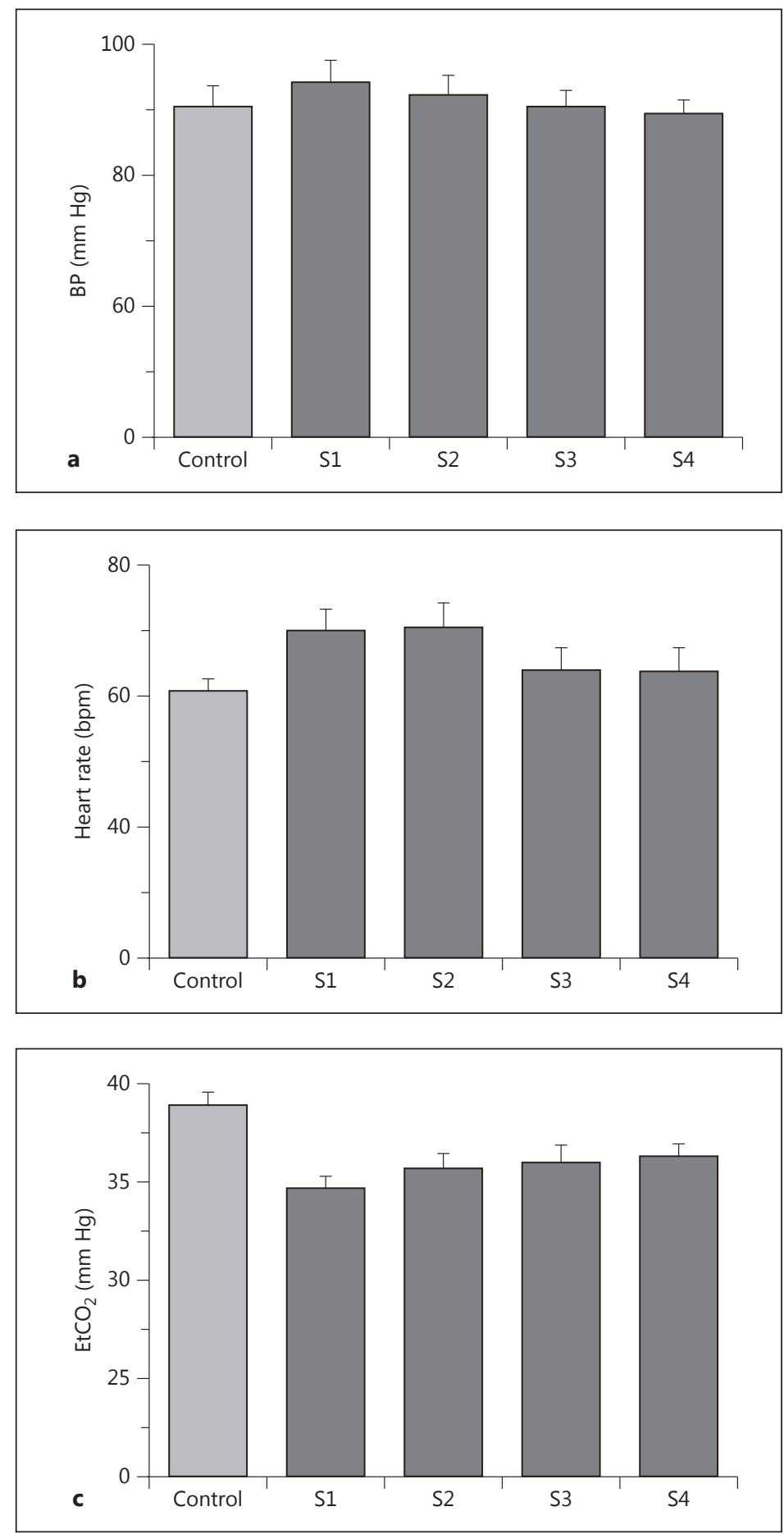

In regions of poor perfusion pressure, arterioles are dilated and thus resistance is low; this results in impaired cerebrovascular reactivity (CVR) in these territories [36]. CVR was not assessed directly in this study, but our previous study used a two-parameter model to express the passive relationship between $\mathrm{BP}$ and $\mathrm{CBFv}$ comprised of critical closing pressure and resistance area product in the acute phase of stroke [37]. Critical closing pressure, which 
under certain conditions can represent the metabolic component of CA, was not affected after stroke, whilst resistance area product, which possibly represents the myogenic response, was impaired [37-39]. Therefore, the smaller CBFv response to neural activation in the acute phase and, as we may speculate, in the subacute phase as well could be (partially) attributed to a 'sluggish' vasodilatory response of the local vessels, as the vascular reserve was diminished but not entirely absent.

\section{Physiological and Clinical Considerations}

The reason(s) for the bilateral impairment of CBF regulation, which seems to be limited to the acute and subacute phases, is (are) not clear. This phenomenon could be explained by arteriolar dysfunction that develops at the ischaemic site and spreads to remote areas later in the post-stroke phase [1]. It was hypothesized that a vicious circle could start in the periinfarct area by spreading local acidosis, and this is amplified by reperfusion (either spontaneously or induced by thrombolysis) with consequent dysautoregulation [40]. In addition, bursts of oxidative stress induced by cerebral ischaemia lead to profound alterations in cerebrovascular regulation. In particular, reactive oxygen species can impair endothelial NO-mediated responses, vasodilation (mediated by $\mathrm{K}^{+}$channel activation), and vasoconstrictor mechanisms [41]. Moreover, pre-existing endothelial dysfunction (due to ageing, internal carotid artery stenosis or hypertension), which may be exacerbated within the acute phase shortly after cortical ischaemic stroke [42], when inflammatory or autonomic changes additionally affect the cerebral vasculature, also need to be considered. An additional explanation relates to transhemispheric diaschisis, where CBF changes contralateral to the side of the ischaemic insult have been widely reported in the animal model and human literature. These studies suggest that CBF changes depend on the magnitude of the ischaemic insult, and gradually tend to return towards the baseline level more than a week after the ischaemic insult [43].

Adding to the complexity of the interpretation of post-stroke cerebral haemodynamic regulation, most patients showed a marked BP increase in all sessions compared to control subjects, though only 5 had a premorbid history of hypertension. Biochemical and structural changes triggered by sustained hypertension can also induce dysfunction of cerebral haemodynamic regulation [44]. Therefore, the sustained hypertension may have contributed to the CA deterioration in the subacute phase.

The evidence of NVC and CA impairment after ischaemic stroke demonstrated in this study and the failure of the myogenic component of CBF regulation in the acute phase shown in a previous study [24] might have implications for therapies directed at improvement of CBF regulation and perfusion. First, the role of BP becomes particularly important whenever a large volume of hypoperfused but still viable tissue, susceptible to changes in systemic BP, is present. The longitudinal findings revealed a deterioration of CA in the subacute phase being restored in the chronic phase. This adds to the debate on the acute use of antihypertensive therapy after stroke [45], particularly very intense BP lowering in all stroke subjects. Secondly, a randomized study demonstrated that aerobic treadmill training improves CVR in chronic stroke patients [46], which could also improve CA and NVC. Very few studies have investigated the evidence that exercise enhances CVR and to our knowledge, this hypothesis has not been tested in the earlier stages of stroke. In healthy subjects, exercise (hand grip and cycling) does not impair CA [47], but CA was affected during heavy (exhaustive) exercise [48]. While the state of CBF regulation after stroke is not yet fully understood, very early mobilization should be undertaken with caution, as an increase in BP in the presence of impaired CA and NVC may bring potential harm to the recovering brain, since CBF regulation might already be impaired and worsened during therapy leading to adverse events. 


\section{Limitations of the Study}

There are several limitations of the current study. The small number of patients reduced statistical power. More than half of the recruited stroke participants withdrew from the study after the first session mainly for reasons unrelated to the research protocol, such as being discharged to rehabilitation $(n=7)$ or undergoing carotid endarterectomy $(n=2)$. A range of circumstances further reduced the number of participants, such as the absence of temporal windows $(n=3)$. The protocol restrictions, particularly related to atrial fibrillation and survival to 3 months, imply that a population with mild-to-moderate stroke severity was recruited, as evidenced by the National Institutes of Health Stroke Scale scores. Therefore, these findings may not be generalizable to a more heterogeneous stroke population, meaning that an interesting hypothesis of the potential influence of stroke subtype could not be adequately explored. Moreover, it was not possible to assess the relevance of stroke sensorimotor deficits at around 2 weeks, because most cases recovered relatively well. Therefore, the longitudinal evolution of CBFv regulation for more severe stroke cases, with poor or limited recovery, still needs to be addressed.

The relatively small group of patients assessed did not allow us to distinguish between right and left hemisphere strokes, although the influence of hemisphere dominance on the CBF response is still not fully understood. Further studies are needed to perform separate analyses of patients with right and left lesions, which may be important given reported hemisphere predominance in autonomic control. In addition, a third of the studied stroke participants had a history of hypertension, whereas the control participants were all normotensive. Future studies will benefit from a second control group composed of non-stroke hypertensive subjects, in order to determine the separate contributions of stroke and hypertension.

There are also a number of acknowledged limitations associated with the use of TCD technology in the assessment of cerebral haemodynamics. First, there is a need for a transtemporal bone window, which is often compromised by age and/or bone thickening of the skull. Secondly, it only provides an assessment of global, usually MCA, hemispheric haemodynamic parameters, where focal changes, especially in the ischaemic penumbra, may be more informative following acute ischaemic stroke. Finally, TCD assesses CBFv, so any implied changes in CBF are consequent on a stable MCA diameter.

\section{Conclusion}

The follow-up of a stroke population over a 3-month period, based on 4 sequential assessments of CA and NVC, showed that both mechanisms of CBFv regulation are impaired at some point during the first weeks after stroke onset, but return to control levels within 3 months. These findings are relevant to guide the timing of interventions to manipulate $\mathrm{BP}$, and potentially for the impact of intensive rehabilitation strategies that may precipitate acute physiological perturbations, but require further exploration in a larger population that better reflects the heterogeneity of stroke, and will also enable the influence of stroke subtype to be investigated.

\section{Acknowledgement}

Angela Salinet was funded by the Brazilian Ministry of Education (grant number 041110-8). 


\section{Cerebrovascular \\ Diseases}

\section{Disclosure Statement}

The authors declare that they have no conflicts of interest.

\begin{tabular}{l|l}
\hline \multicolumn{2}{l}{ Cerebrovasc Dis Extra 2014;4:186-197 } \\
\hline DOI: $10.1159 / 000366017$ & $\begin{array}{l}\text { ( ) 2014 S. Karger AG, Basel } \\
\text { www.karger.com/cee }\end{array}$ \\
\hline
\end{tabular}

Salinet et al.: The Longitudinal Evolution of Cerebral Blood Flow Regulation after Acute Ischaemic Stroke

\section{References}

1 Attwell D, Buchan AM, Charpak S, Lauritzen M, MacVicar BA, Newman EA: Glial and neuronal control of brain blood flow. Nature 2010;468:232-243.

2 Attwell D, Iadecola C: The neural basis of functional brain imaging signals. Trends Neurosci 2002;25:621-625.

-3 Donahue MJ, Stevens RD, de Boorder M, Pekar JJ, Hendrikse J, van Zijl PCM: Hemodynamic changes after visual stimulation and breath holding provide evidence for an uncoupling of cerebral blood flow and volume from oxygen metabolism. J Cereb Blood Flow Metab 2009;29:176-185.

-4 Aries MJH, Elting JW, De Keyser J, Kremer BPH, Vroomen PCAJ: Cerebral autoregulation in stroke. Stroke 2010; 41:2697-2704.

5 Salinet ASM, Haunton V, Panerai R, Robinson T: A systematic review of cerebral hemodynamic responses to neural activation following stroke. J Neurol 2013;260:2715-2721.

-6 Reinhard M, Rutsch S, Lambeck J, Wihler C, Czosnyka M, Weiller C, Hetzel A: Dynamic cerebral autoregulation associates with infarct size and outcome after ischemic stroke. Acta Neurol Scand 2011;125:156-162.

7 Calautti C, Baron JC: Functional neuroimaging studies of motor recovery after stroke in adults - a review. Stroke 2003;34:1553-1566.

-8 Altamura C, Reinhard M, Vry MS, Kaller CP, Hamzei F, Vernieri F, Rossini PM, Hetzel A, Weiller C, Saur D: The longitudinal changes of BOLD response and cerebral hemodynamics from acute to subacute stroke. A fMRI and TCD study. BMC Neurosci 2009;10:151.

-9 Askim T, Indredavik B, Vangberg T, Haberg A: Motor network changes associated with successful motor skill relearning after acute ischemic stroke: a longitudinal functional magnetic resonance imaging study. Neurorehabil Neural Repair 2009;23:295-304.

10 Calautti C, Leroy F, Guincestre JY, Baron JC: Dynamics of motor network overactivation after striatocapsular stroke - a longitudinal PET study using a fixed-performance paradigm. Stroke 2001;32:2534-2542.

11 Carey LM, Abbott DF, Egan GF, Bernhardt J, Donnan GA: Motor impairment and recovery in the upper limb after stroke - behavioral and neuroanatomical correlates. Stroke 2005;36:625-629.

12 Cuadrado ML, Egido JA, Gonzalez-Gutierrez JL, Varela-de-Seijas E: Bihemispheric contribution to motor recovery after stroke: a longitudinal study with transcranial Doppler ultrasonography. Cerebrovasc Dis 1999; 9:337-344.

13 Dawson S, Panerai R, Potter J: Serial changes in static and dynamic cerebral autoregulation after acute ischaemic stroke. Cerebrovasc Dis 2003;16:69-75.

14 Nelles G, Jentzen W, Bockisch A, Diener HC: Neural substrates of good and poor recovery after hemiplegic stroke: a serial PET study. J Neurol 2011;258:2168-2175.

15 Kuptniratsaikul V, Kovindha A, Piravej K, Dajpratham P: First-year outcomes after stroke rehabilitation: a multicenter study in Thailand. ISRN Rehabil 2013;2013:1-6.

16 Oldfield R: The assessment and analysis of handedness: the Edinburgh inventory. Neuropsychologia 1971;9: 97-113.

17 Lyden P, Brott T, Tilley B, Welch KMA, Mascha EJ, Levine S, Haley EC, Grotta J, Marler J: Improved reliability of the NIH stroke scale using video training. Stroke 1994;25:2220-2226.

18 van Swieten JC, Koudstaal PJ, Visser MC, Schouten HJ, van Gijn J: Interobserver agreement for the assessment of handicap in stroke patients. Stroke 1988;19:604-607.

19 Salinet ASM, Robinson TG, Panerai RB: Reproducibility of cerebral and peripheral haemodynamic responses to active, passive and motor imagery paradigms in older healthy volunteers: afTCD study. J Neurosci Methods 2012;206:143-150.

20 Salinet ASM, Panerai RB, Robinson TG: Effects of active, passive and motor imagery paradigms on cerebral and peripheral hemodynamics in older volunteers: a functional TCD study. Ultrasound Med Biol 2012;38:997-1003.

-21 Salinet ASM, Robinson TG, Panerai RB: Cerebral blood flow response to neural activation after acute ischaemic stroke: a failure of myogenic regulation? J Neurol 2013;260:2588-2595.

22 Katsogridakis E, Bush G, Fan L, Birch AA, Simpson DM, Allen R, Potter JF, Panerai RB: Detection of impaired cerebral autoregulation improves by increasing arterial blood pressure variability. J Cereb Blood Flow Metab 2013;33:519-523.

23 Tiecks FP, Lam AM, Aaslid R, Newell DW: Comparison of static and dynamic cerebral autoregulation measurements. Stroke 1995;26:1014-1019.

24 Bamford J, Sandercock P, Dennis M, Warlow C, Burn J: Classification and natural history of clinically identifiable subtypes of cerebral infarction. Lancet 1991;337:1521-1526.

25 Dijkhuizen RM, Ren J, Mandeville JB, Wu O, Ozdag FM, Moskowitz MA, Rosen BR, Finklestein SP: Functional magnetic resonance imaging of reorganization in rat brain after stroke. Proc Natl Acad Sci USA 2001;98: 12766-12771. 
Salinet et al.: The Longitudinal Evolution of Cerebral Blood Flow Regulation after Acute Ischaemic Stroke

$>26$ during recovery from corticospinal tract infarction. Stroke 2000;31:656-661.

Nelles G, Spiekermann G, Jueptner M, Leonhardt G, Muller S, Gerhard H, Diener HC: Reorganization of sensory and motor systems in hemiplegic stroke patients - a positron emission tomography study. Stroke 1999;30: 1510-1516.

28 Carey LM, Abbott DF, Egan GF, O’Keefe GJ, Jackson GD, Bernhardt J, Donnan GA: Evolution of brain activation with good and poor motor recovery after stroke. Neurorehabil Neural Repair 2006;20:24-41.

29 Silvestrini M, Caltagirone C, Cupini LM, Matteis M, Troisi E, Bernardi G: Activation of healthy hemisphere in poststroke recovery - a transcranial Doppler study. Stroke 1993;24:1673-1677.

30 Weiller C, Chollet F, Friston K, Wise R, Frackowiak R: Functional reorganization of the brain in recovery from striatocapsular infarction in man. Ann Neurol 1992;31:463-472.

31 Silvestrini M, Troisi E, Matteis M, Razzano C, Caltagirone C: Correlations of flow velocity changes during mental activity and recovery from aphasia in ischemic stroke. Neurology 1998;50:191-195.

-32 Mimura M, Kato M, Kato M, Sano Y, Kojima T, Naeser M, Kashima H: Prospective and retrospective studies of recovery in aphasia. Changes in cerebral blood flow and language functions. Brain 1998;121:2083-2094.

-33 Dawson SL, Blake MJ, Panerai RB, Potter JF: Dynamic but not static cerebral autoregulation is impaired in acute ischaemic stroke. Cerebrovasc Dis 2000;10:126-132.

-34 Reinhard M, Neunhoeffer F, Gerds TA, Niesen WD, Buttler KJ, Timmer J, Schmidt B, Czosnyka M, Weiller C, Hetzel A: Secondary decline of cerebral autoregulation is associated with worse outcome after intracerebral hemorrhage. Intensive Care Med 2010;36:264-271.

-35 Panerai RB: Assessment of cerebral pressure autoregulation in humans - a review of measurement methods. Physiol Meas 1998;19:305-338.

36 Zhao P, Alsop DC, Abduljalil A, Selim M, Lipsitz L, Novak P, Caplan L, Hu K, Novak V: Vasoreactivity and periinfarct hyperintensities in stroke. Neurology 2009;72:643-649.

-37 Panerai RB, Eyre M, Potter JF: Multivariate modeling of cognitive-motor stimulation on neurovascular coupling: transcranial Doppler used to characterize myogenic and metabolic influences. Am J Physiol Regul Integr Comp Physiol 2012;303:R395-R407.

38 Panerai RB: The critical closing pressure of the cerebral circulation. Med Eng Phys 2003;25:621-632.

-39 Panerai RB, Moody M, Eames PJ, Potter JF: Cerebral blood flow velocity during mental activation: interpretation with different models of the passive pressure-velocity relationship. J Appl Physiol 2005;99:23522362.

-40 Dohmen C, Bosche B, Graf R, Reithmeier T, Ernestus RI, Brinker G, Sobesky J, Heiss WD: Identification and clinical impact of impaired cerebrovascular autoregulation in patients with malignant middle cerebral artery infarction. Stroke 2007;38:56-61.

41 Faraci FM: Oxidative stress: the curse that underlies cerebral vascular dysfunction? Stroke 2005;36:186-188.

42 Stevenson SF, Doubal FN, Shuler K, Wardlaw JM: A systematic review of dynamic cerebral and peripheral endothelial function in lacunar stroke versus controls. Stroke 2012;41:e434-e442.

43 Andrews R: Transhemispheric diaschisis. A review and comment. Stroke 1991;22:943-949.

44 Faraci FM, Baumbach GL, Heistad DD: Cerebral circulation: humoral regulation and effects of chronic hypertension. J Am Soc Nephrol 1990;1:53-57.

-45 Ivey FM, Ryan AS, Hafer-Macko CE, Macko RF: Improved cerebral vasomotor reactivity after exercise training in hemiparetic stroke survivors. Stroke 2011;42:1994-2000.

46 Sandset EC, Murray GD, Bath PMW, Kjeldsen SE, Berge E: Relation between change in blood pressure in acute stroke and risk of early adverse events and poor outcome. Stroke 2012;43:2108-2114.

47 Ogoh S, Sato K, Akimoto T, Oue A, Hirasawa A, Sadamoto T: Dynamic cerebral autoregulation during and after handgrip exercise in humans. J Appl Physiol 2010;108:1701-1705.

-48 Ogoh S, Dalsgaard MK, Yoshiga CC, Dawson EA, Keller DM, Raven PB, Secher NH: Dynamic cerebral autoregulation during exhaustive exercise in humans. Am J Physiol Heart Circ Physiol 2005;288:H1461-H1467. 